\title{
Time Dependence of Chemical Freeze-out in Relativistic Heavy Ion Collisions
}

\author{
R. Bellwied ${ }^{1}$, H. Caines ${ }^{2}$, and T.J. Humanic ${ }^{2}$ \\ 1 Wayne State University, Physics Department, Detroit, Michigan \\ 2 The Ohio State University, Physics Department, Columbus, Ohio
}

(23-Mar-00, submitted to Phys.Rev.C)

\section{Introduction}

Recent model calculations, which were successfully applied to CERN SPS data, implied that most measured particle distributions in relativistic heavy ion collisions can be described with statistical models [1]. The underlying kinematics requires at least thermal equilibration at freezeout. All kinetic spectra, like the transverse momentum and the rapidity distributions, can be explained with a single freezeout temperature, if one assumes a certain expansion velocity after hadronization. Attempts to describe not only the kinetic spectra but also the particle abundances and particle ratios with a common temperature were successful, but led to a different temperature. Whereas the slopes of all momentum spectra yield a T around $130 \mathrm{MeV}$ after inclusion of the expansion velocity, the particle ratios lead to a $\mathrm{T}$ of about 180 $\mathrm{MeV}$ for a common chemical potential. Two questions arise: is the chemical freezeout decoupled from the thermal freezeout, and is the system chemically equilibrated at chemical freezeout? In a systematic study of measured particle ratios and spectra in the framework of a thermal model, Heinz has shown that for all CERN and AGS data the chemical freezeout seems to be decoupled from the thermal freeze-out [2].

Rafelksi has recently postulated that, on the basis of strange particle ratios measured in $\mathrm{Pb}+\mathrm{Pb}$ at $\mathrm{CERN}$, one has to assume a phase transition from QGP to Hadron Gas, and that at the time of hadronization the system is not in chemical equilibrium [3]. To describe the kinetic spectra and particle yields this model requires that the chemical composition of the fireball remains unchanged between hadronization and thermal freeze-out. That means all interactions during this time interval have to be elastic. This model is in agreement with the fact that all systems from $\mathrm{e}^{+} \mathrm{e}^{-}$collisions up to AA-collisions seem to yield the same chemical freezeout temperature [1]. Thus, the shapes of the particle spectra might change due to rescattering, but the particle abundances and ratios remain constant from hadronization on, a theory which is commonly referred to as 'sudden hadronization'. This process explicitly prohibits hadro-chemical equilibration through rescattering. Only elastic processes 
can be employed to explain the difference between the temperature calculated from particle ratios and the temperature calculated from kinetic particle spectra. Thus, the ratios effectively reflect the temperature at hadronization, whereas the kinetic freezeout temperature describes the actual freezeout (last elastic final state interaction). The fact that the hadronization temperature is about $180 \mathrm{MeV}$, close to the critical temperature, can be viewed as an indicator that the system actually crossed a phase transition. However, the fact that all inelastic scattering processes cease at hadronization time is a very strong constraint. Hadronic population ratios are, in this theory, the result of the hadronization mechanism and not caused by interactions during the hadronic rescattering phase. The main argument employed is that the relaxation times of all relevant hadronic channels is well above $\tau=3 \mathrm{fm} / \mathrm{c}$ at which time the temperature of the system has dropped below $\mathrm{T}=185 \mathrm{MeV}$ which leads to a small probability of chemical equilibration after hadronization. Also based on results of HBT measurements, Stock argues that the time between hadronization and thermal freezeout might simply be too short to develop a significant inelastic cross section contribution [8]. The required time interval for hadronic processes to adjust the strangeness content is long and the chemical rates are small since the production of pairs of strange hadrons carries a large energy penalty factor. In Stock's scenario it takes upward of $3 \mathrm{fm} / \mathrm{c}$ to equilibrate strangeness in the hadronic phase, a time span that, he postulates, is not available owing to the rapid expansion prevailing at hadronization time.

Surprisingly, a partonic cascade approach, which is very different from the model described above, yields results similar to a thermal sudden hadronization theory. Geiger and Ellis [4] have shown that at large incident energies the hadronization process can determine the final particle ratios based on its combined non-perturbative mechanisms and it can even lead to direct chemical equilibrium. The final multihadronic state materializes into maximal entropy (equilibrium) straight out of the partonic phase. Although certain remnants of the initial particle structure functions remain, the hadron yield near midrapidity stems mostly from the initial parton cascade processes. In this theory, the partonic phase exhibits $\epsilon \geq 2 \mathrm{Gev} / \mathrm{fm}^{3}$ until about $2 \mathrm{fm} / \mathrm{c}$. Hadronization occurs after a formation time of about $1 \mathrm{fm} / \mathrm{c}$ and a mixed phase of hadrons and partons ends at $\tau \approx 20 \mathrm{fm} / \mathrm{c}$, when $\epsilon$ is less than $0.2 \mathrm{GeV} / \mathrm{fm}^{3}$. Although the concept of sudden hadronization is lost in this theory, due to the required long mixed phase between partons and hadrons, the results are comparable to a thermal model without inelastic final state interactions. While the parton models require a mixed phase for the proper kinematic expansion of the system, they do not require any final state interactions to describe the measured particle abundances. Kapusta and Mekjian [7] derived estimates for the dynamical equilibration (relaxation) times of quark flavors in a quark gluon gas and deduced predictions for several equilibrium abundance ratios. These ratios are in good agreement with the data, which led Stock [8] to postulate that the equilibrated ratios formed in pre-hadronization reactions do not change during the final state interactions. 
The main motivation for this paper is the fact that most dynamic transport codes, which in the past were successfully applied to describe data from CERN and the AGS, behave very differently from either the parton cascade or the thermal sudden hadronization approach. These models employ measured inelastic scattering cross sections for different particle species to describe the hadronic transport from hadronization to kinetic freezeout. Many of the relevant particle species are susceptible to number changing interactions, in particular inelastic meson interactions lead to sizable contributions to the hyperon production cross section. Both, strangeness creation interactions (e.g. $\pi \mathrm{N} \rightarrow \mathrm{K} \Lambda$ ) and strangeness exchange interactions (e.g. $\mathrm{KN} \rightarrow \pi \Lambda$ ) can be exothermic and should affect strange particle production.

Pratt and Haglin have recently pointed out that number changing cross sections after hadronization seem to be relevant for the description of the pion abundance at CERN [5]. They have shown that hadrons interact several times before the freezeout temperatures are reached if one assumes that binary modeling starts at $\mathrm{T}=160 \mathrm{MeV}$ in a chemically and thermally equilibrated system. It is interesting to note that the chemical equilibration times for strange and non strange quarks shown by Stock and Pratt/Haglin are very comparable. An independent study by Humanic, using an early version of the transport code which is the basis of this paper, shows that pions interact on average ten times between hadronization and freezeout [9]. The time from hadronization to freezeout is quite long (about $15 \mathrm{fm} / \mathrm{c}$ ) in particular for the lightest mesons. If these transport models are correct then we should be able to simulate a quantitative dynamic evolution of all particle abundances through the rescattering phase.

\section{Hadronic particle ratio simulations}

In the following we attempt to prove that in particular the abundance of the singly strange baryon, the $\Lambda$, is affected by a series of inelastic rescattering processes by the time thermal freeze-out is accomplished. We have employed the dynamic transport code described in Ref.[9] to model the initial state of the system at hadronization and the subsequent hadronic rescattering to freeze out. This code has been shown to well represent the features and dynamic dependences of the transverse mass spectra and HBT observables for CERN SPS $\mathrm{Pb}+\mathrm{Pb}$ data [9]. In addition to the already implemented cross sections for pions, kaons, nucleons and their associated resonances, we have augmented the code to include elastic and inelastic rescattering of $\Lambda$ baryons. The initial conditions are described by a common temperature and spatial extension. All particles hadronize at a proper time of $1 \mathrm{fm} / \mathrm{c}$. A Bjorken-type geometry was used to simulate the dynamic evolution of the fireball from hadronization time. No initial radial flow is needed for the calculations to agree with data [9].

Fig. 1 shows the effect of rescattering on all four relevant particle abundances $(\pi$, $\mathrm{K}$, nucleons, and $\Lambda$ ). 

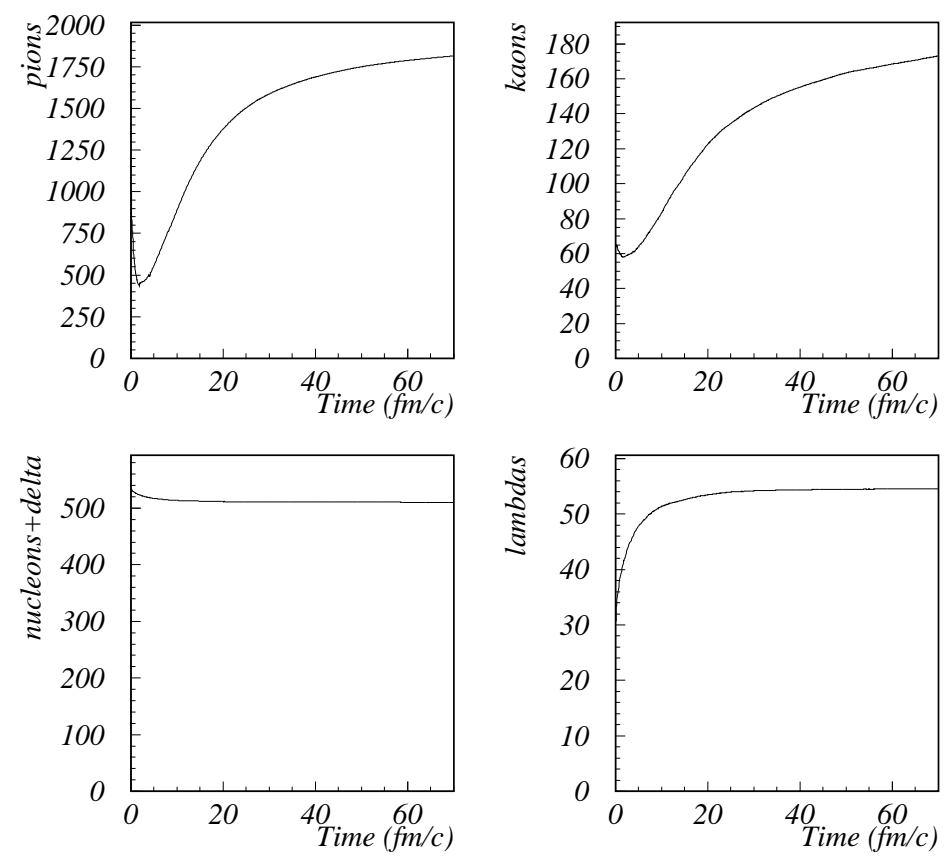

Figure 1: Particle abundances as a function of time between hadronization and kinematic freeze-out

In the case of the nucleons, the nucleon resonance production and subsequent decay leads to no change in the number of nucleons and is thus considered an elastic process, whereas in the pion case, generation from resonance decay is considered a number changing process (e.g. $\mathrm{N} \pi \rightarrow \Delta \pi \rightarrow \mathrm{N} \pi \pi$ ). The same is true for vector meson production $(\rho, \phi, \omega)$ and decay into pions. Fig.1 shows that the nucleon abundance is close to constant, but the meson and strange baryon abundances display a steady increase. One of the large contributing elastic reactions is $\pi \pi \rightarrow \rho \rightarrow \pi \pi$ which is fast and keeps the number of $\rho$ 's in equilibrium but does not change the pion number. Reactions that change the overall pion number like $\pi \pi \rightarrow \eta^{\prime} \rightarrow \pi \pi \pi$ are generally slower and thus do not have a large effect on the chemical state formed at hadronization. In addition reactions that conserve the net number of strange quarks occur rapidly, e.g. $\mathrm{KN} \rightarrow \Lambda \pi$, but reactions that change the number of strange quarks, e.g. $\pi \mathrm{N} \rightarrow \mathrm{K} \Lambda$, are slow as they require a strange and an antistrange hadron to either interact or to be produced jointly.

Fig.2 shows the relative contributions of all relevant reaction channels to the $\Lambda$ abundance as a function of time for the $\mathrm{Pb}+\mathrm{Pb}$ and the $\mathrm{S}+\mathrm{S}$ systems at the SPS. In the $\mathrm{Pb}$ case the direct $\Lambda$ production at hadronization yields only around $50 \%$ $(\approx 30 \Lambda$ 's $)$ of the total yield at kinetic freezeout. It is apparent that $\Lambda$ generation 
processes dominate the $\Lambda$ inelastic re-scattering, which effectively leads to a factor two enhancement in $\Lambda$ production well after hadronization. In the smaller $\mathrm{S}+\mathrm{S}$ system the rescattering contributes only about $10 \%$ of the total $\Lambda$ yield.
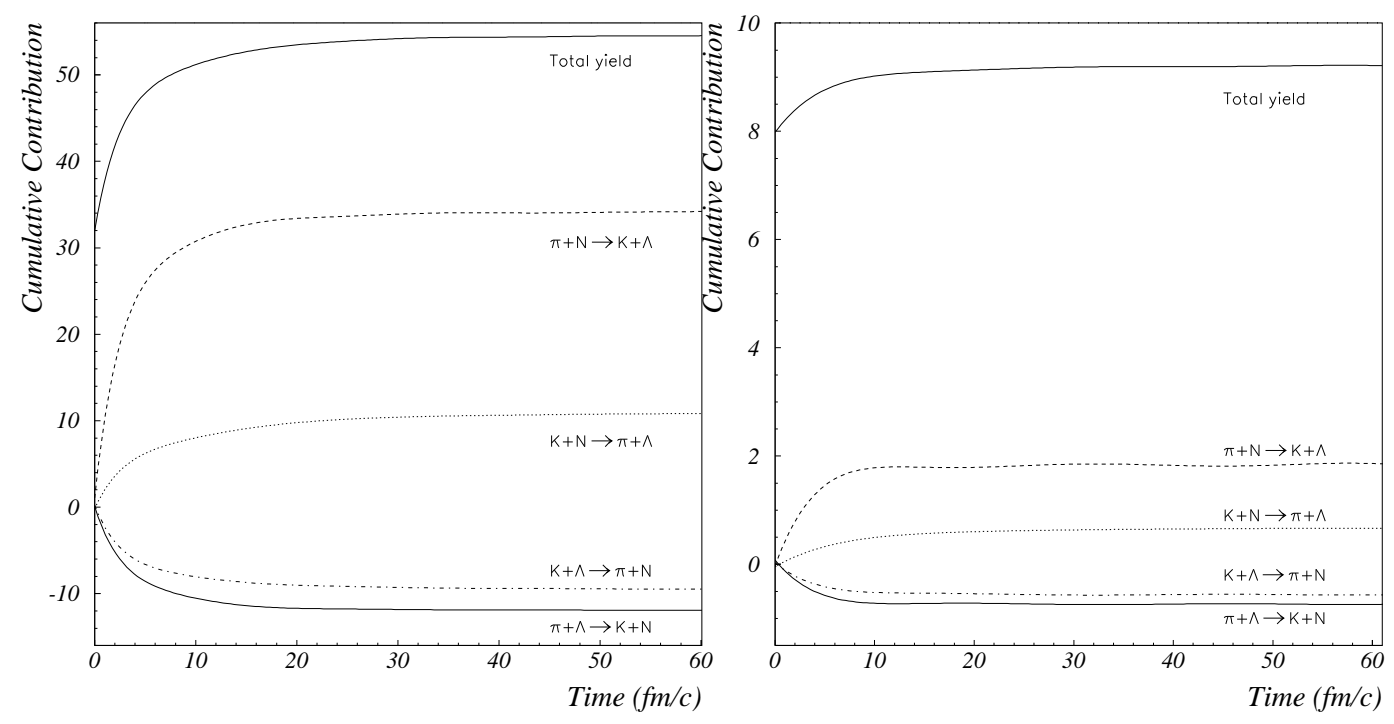

Figure 2: Contributing channels to the $\Lambda$ cross section in a.) $\mathrm{Pb}+\mathrm{Pb}$ and b.) $\mathrm{S}+\mathrm{S}$

The plots also show that $\Lambda$ production and annihilation through inelastic rescattering stops after a certain time (chemical freezeout). It turns out, though, that this is not due to our model reaching strangeness chemical equilibrium but rather based on the rapid expansion and the resulting drop in particle density. After around $30 \mathrm{fm} / \mathrm{c}$ the particle density is simply too small to support further inelastic scatterings which produce strange baryons. To prove that our model reaches equilibrium for the case of a non-expanding system by properly envoking detailed balance in our cross sections, we chose a fixed maximum radius of $10 \mathrm{fm}$ and plotted the $\Lambda$ production and annihilation channels as a function of time. From Fig.3 it is apparent that equilibrium is reached, the total $\Lambda$ yield becomes constant, whereas the production and annihilation yields continue to rise at long times. Thus chemical equilibration is possible if the expansion velocity is sufficiently small. Our calculations agree with other thermal model calculations, though, in predicting that the $\mathrm{Pb}+\mathrm{Pb}$ system at CERN SPS energies does not reach chemical equilibrium before freeze-out. Based on Fig.1 we can conclude that all particle ratios that include the $\Lambda$ yield will undergo significant changes during rescattering, a fact which is incompatible with the notion of sudden hadronization. If we force sudden hadronization in our code by simply turning off all inelastic rescattering modes, our model calculations lead to a much smaller HBT radius in both the $\pi \pi$ and $\mathrm{KK}$ channels compared to the actually measured radii. 


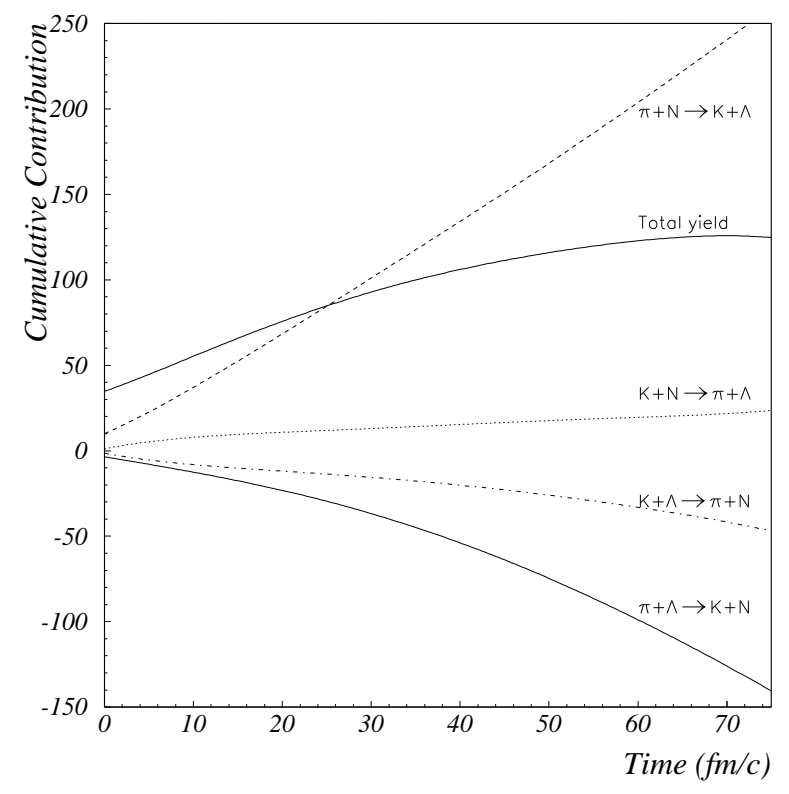

Figure 3: Detailed balance in a system of fixed maximum radius $(\mathrm{r}=10 \mathrm{fm})$

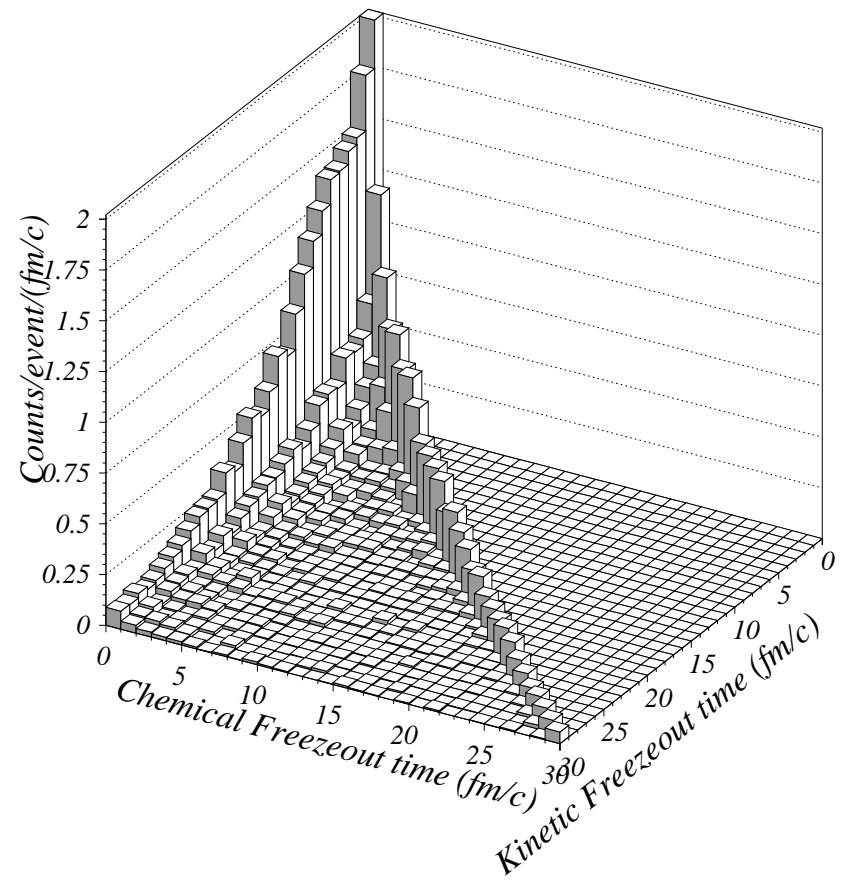

Figure 4: Relation between chemical freezeout time and kinematic freezeout time for measurable $\Lambda$ particles 
Fig. 4 shows the relation between kinetic and chemical freezeout time for every $\Lambda$ measurable in the $\mathrm{Pb}+\mathrm{Pb}$ system after kinetic freezeout. It is apparent that only about half of the emitted $\Lambda$ 's show a chemical freezeout time consistent with the hadronization time, which is required for sudden hadronization $\left(\mathrm{t}_{\text {chem }}=0, \mathrm{t}_{\text {kin }}=\right.$ any $)$. Many $\Lambda$ 's (about $25 \%$ ) are actually produced in their final rescattering step $\left(\mathrm{t}_{\text {chem }}=\mathrm{t}_{\text {kin }}\right)$.

A comparison of the one-dimensional freeze-out time distributions for the measured $\Lambda$ 's in Fig.5 shows that the chemical freeze-out occurs fast but it is not 'sudden'. $90 \%$ of the $\Lambda$ 's are chemically frozen out after $5 \mathrm{fm} / \mathrm{c}$, but of those about $40 \%$ freezeout after hadronization. The according kinetic freeze-out times are shown in Fig.6. Fig.6a shows the difference in kinetic freeze-out time between the direct and the produced $\Lambda$ component. The production after rescattering significantly enhances the average freeze-out time and alters the shape of the distribution. But the $\Lambda$ freeze-out is still peaked significantly earlier than the pion and proton freeze-out in our model [9] as seen in Fig.6b. The main reason is that the inelastic and elastic cross sections for $\Lambda$ induced reactions are much smaller than the proton or pion interaction cross sections. This trend will continue for multi-strange baryons and it should lead to a very early decoupling of the $\Omega$ from the fireball [14]. For multi-strange baryons the notion of sudden hadronization seems thus more sensible.

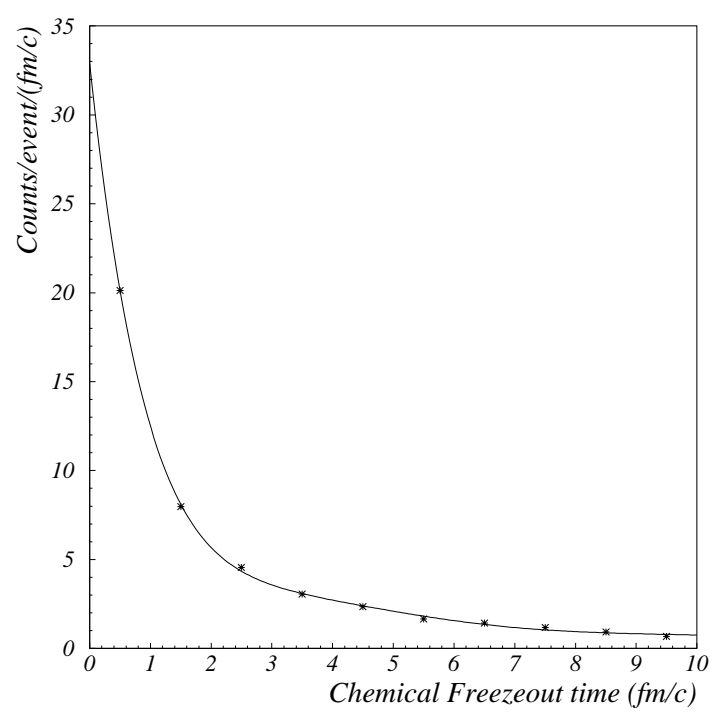

Figure 5: Chemical freezeout time for measurable $\Lambda$ particles.

Fig.7 shows the transverse mass spectrum of the frozen out $\Lambda$ 's. The model is in perfect agreement with the data measured by NA49 as it is for the pions and nucleons. 

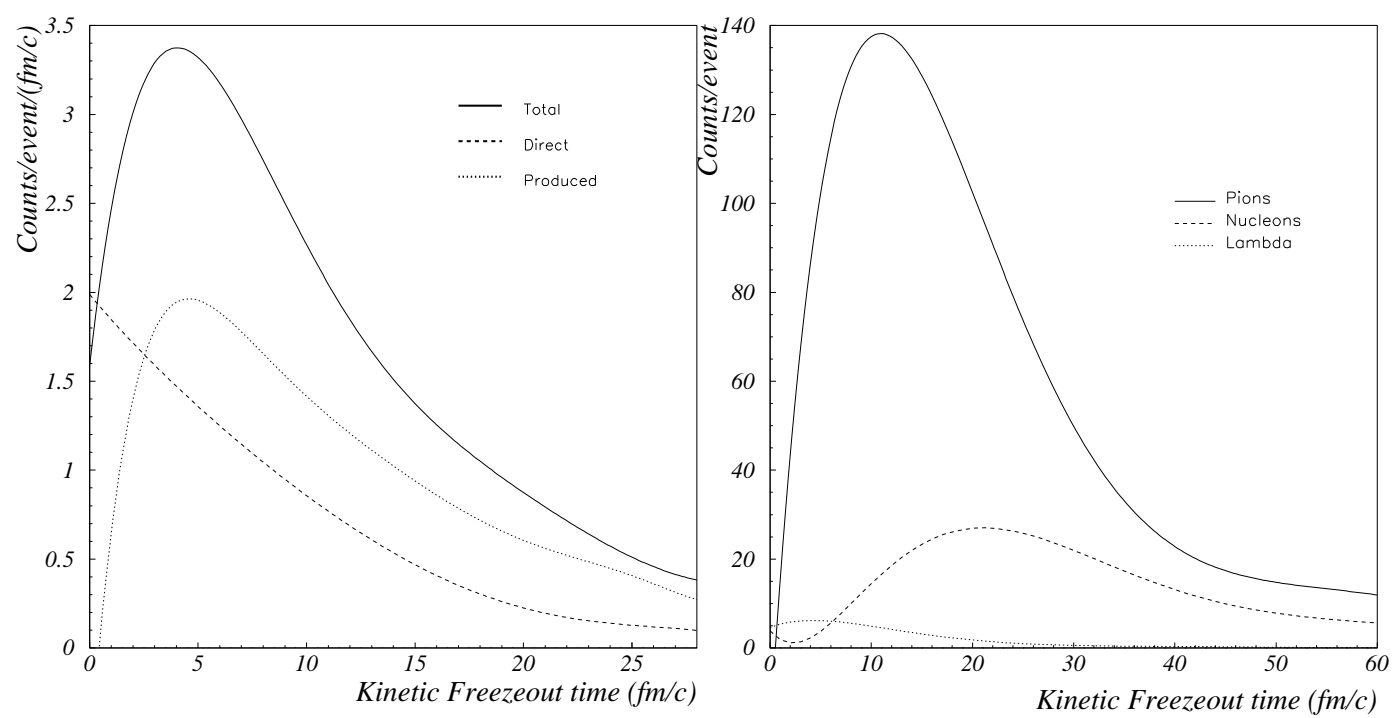

Figure 6: a.) Kinetic freezeout time for the different components of the $\Lambda$ spectrum, b.) Comparison between the kinetic freezeout time of measurable $\Lambda$ 's , $\pi$ 's and protons

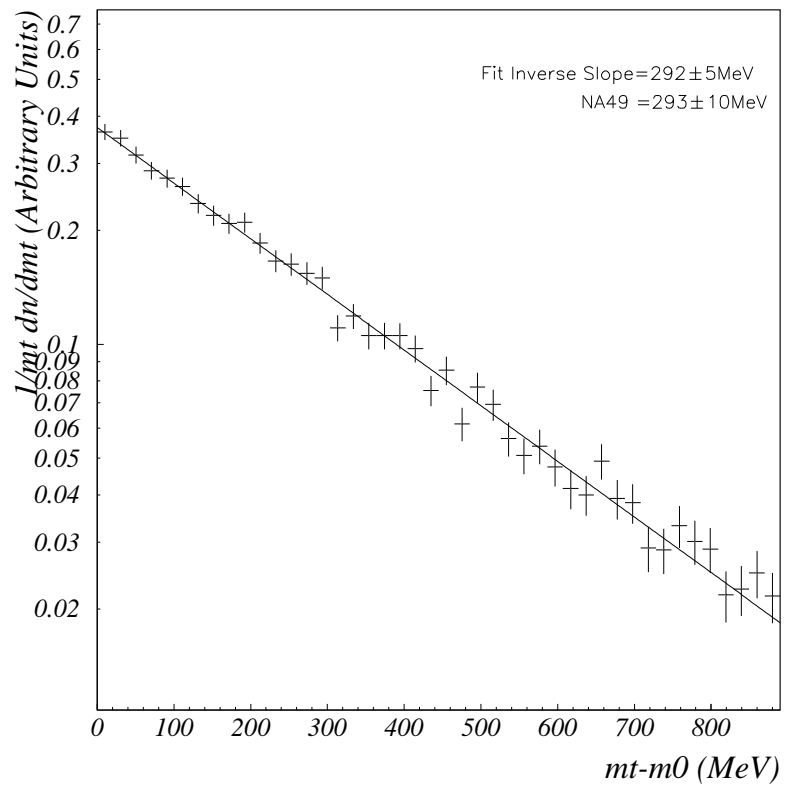

Figure 7: Transverse mass spectrum for $\Lambda$ from our calculations. The extracted slope parameter is seen to agree with data (NA49). 


\section{Conclusions}

Based on our calculations we conclude that neither the $\Lambda$ yield nor any particle ratio including $\Lambda$ 's are suitable thermometers for the hadronization temperature. These measurements may still be indirect QGP indicators in any transport code description, though, simply through determination of the required initial hadronization conditions (initial temperature and energy density). This is a departure from the original wisdom that only leptonic probes can act as real indicators of the initial conditions.

We have shown that the $\Lambda$ abundance is strongly affected by final state interactions and is not reproducable under the condition of sudden hadronization, if the model attempts to describe all hadronic measurements in particular in the heaviest SPS systems. The probability of inelastic scattering is sufficiently large that even during the rather short time lapse between hadronization and thermal freezeout the yields change considerably. Thus, conclusions drawn from the strange baryon ratios at CERN concerning a QGP phase transition are probably an oversimplification regarding the actual interactions inside the fireball.

Our simulations agree with other thermal models that the chemical properties are frozen well before the kinetic spectra but we also demonstrate that hadronization and chemical freezeout mostly do not coincide for strange baryons produced in $\mathrm{Pb}+\mathrm{Pb}$. Thus the hadronization should occur above $\mathrm{T}=180 \mathrm{MeV}$ which was deduced from the ratios as the temperature for chemical freezeout. Indeed, in our calculation the system hadronizes at $213 \mathrm{MeV}$, well above the critical temperature. It is also evident that both, the $\Lambda$ chemical and thermal freeze-out, occur much earlier than the proton and pion freeze-out. Recent detailed simulations of multi-strange baryon ratios and spectra, seem to indicate that this early decoupling follows a trend as a function of the strangeness content. This effect can be attributed to differences in the rescattering probabilities [13]. In particular the apparent lack of flow in the $\Omega$ transverse momentum spectrum could be correlated with the very high $\Omega$ particle ratios. Both point at a uniquely different production mechanism for the $\Omega$. In Dumitru's calculations the $\Omega$ 's undergo on average two collisions after hadronization, whereas the number increases to 3.5 and 5 for $\Xi$ and $\Lambda$, respectively [13]. This early decoupling of the $\Omega$ led van Hecke et al. to postulate that this is the reason for the lower emission temperatures for $\Omega$ 's as measured at the SPS [14. Our calculation indicates that even the $\Lambda$ thermally freezes out after a few $\mathrm{fm} / \mathrm{c}$, due the rather small elastic scattering cross section during the rescattering phase. It is our goal to extend our transport code to include the multiply strange baryons to determine more quantitatively the dynamic differences between strange and multistrange baryons.

The complete measurement of strange particle production at RHIC, from the Kaon to the $\Omega$, will be an important exercise. Our SPS study shows that final state interactions have to taken into account in order to properly describe singly strange particle production. Thus, Kaon and $\Lambda$ measurements alone might not lead to conclusive proof of the formation of a QGP. However plans for detailed measurements 
of strangeness production up to and including the $\Omega$ are well underway at RHIC and we are looking forward to this exciting new era in relativistic heavy ion physics.

\section{References}

[1] F. Becattini, J.Phys. G23 (1997) 1933

[2] U. Heinz, Nucl. Phys. A661 (1999) 140c and J.Phys. G25 (1999) 263

[3] J. Rafelski, J.Phys. G25 (1999) 295

[4] K. Geiger and J. Ellis,Phys. Rev. D54 (1996) 949

[5] S. Pratt and K. Haglin, Phys. Rev. C59 (1999) 3304

[6] S. Margetis et al. (NA49 Collab), J. Phys. G25 (1999) 189

[7] J. Kapusta and A.Z. Mekjian, Phys. Rev. D33 (1986) 1304

[8] H. Stock, Phys.Lett. B456 (1999) 277

[9] T. Humanic, Phys. Rev. C57 (1998) 866

[10] M. Prakash et al. Phys. Rep. 227 (1993) 321

[11] M.C. Abreu et al. (NA50 Collab.), Phys. Lett. B450 (1999) 456

[12] F. Karsch et al., Z. Phys. 37 (1988) 617

[13] A. Dumitru et al., Phys.Lett. B460 (1999) 411

[14] H. van Hecke et al., Phys. Rev. Lett. 81 (1998) 5264 